\title{
A U6 snRNA:pre-mRNA interaction can be rate-limiting for $\mathrm{U} 1$-independent splicing
}

\author{
John D. Crispino and Phillip A. Sharp ${ }^{1}$ \\ Massachusetts Institute of Technology, Center for Cancer Research and Department of Biology, Cambridge, Massachusetts \\ 02139-4307 USA
}

The full set of consensus sequences at the 5' splice site is recognized during splicing of pre-mRNA in extracts depleted of U1 snRNP. High concentrations of HeLa SR proteins or purified SC35 alone promote the splicing of specific RNA substrates, bypassing the requirement for U1 snRNP in formation of the U2 snRNP-pre-mRNA complex. Under these conditions, mutations in the substrate that increase the sequence complementarity between U6 snRNA and the 5' splice site region can facilitate splicing. This provides additional strong evidence that U1 snRNP is not essential for splicing. Thus, the consensus sequence at the $5^{\prime}$ splice site is probably recognized twice during splicing of most introns; however, some pre-mRNAs could potentially be processed in the absence of interactions with U1 snRNP in regions of the nucleus containing high concentrations of SR proteins.

[Key Words: Pre-mRNA splicing; SR proteins; 5' splice site recognition; U1 snRNP]

Received June 6, 1995; revised version accepted August 8, 1995.

Pre-mRNA splicing occurs within a large multisubunit complex termed the spliceosome. The spliceosome, which is composed of four snRNP particles (U1, U2, U4) U6, and U5) and many non-snRNP protein factors, forms in a stepwise manner on the substrate (for review, see Moore et al. 1993; Madhani and Guthrie 1994). In the first commitment step, Ul snRNP, in conjunction with the splicing factor U2AF and several members of the SR protein family, associates with the pre-mRNA to form the E complex (Michaud and Reed 1993; Staknis and Reed 1994). During this reaction, U1 snRNP binds to the $5^{\prime}$ splice site by base pairing (Zhuang and Weiner 1986; Séraphin et al. 1988; Siliciano and Guthrie 1988) and promotes the binding of U2 snRNP to the branch region, forming the A complex (Konarska and Sharp 1986, 1987; Séraphin and Rosbash 1989; Barabino et al. 1990; Michaud and Reed 1991). Finally, the complete spliceosome is formed by the addition of the U4/U5/U6 trisnRNP.

Several rearrangements occur within the spliceosome before the first covalent reaction resulting in the cleavage of the 5' splice site and branch formation. First, U4 snRNA is separated from the U6 snRNA so that U2 and U6 can interact; these two RNAs are proposed to form the catalytic center (Madhani and Guthrie 1992; McPheeters and Abelson 1992). In addition, the U1 snRNA is probably displaced from the 5' splice site (Konforti et al. 1993), whereas U6 snRNA binds to distal nucleotides in the $5^{\prime}$ splice site $(+4$ through +6$)$. The two RNAs can be cross-linked in this region by UV irradia-

${ }^{1}$ Corresponding author. tion (Sawa and Abelson 1992; Sontheimer and Steitz 1993|. Furthermore, allele-specific complementation between mutations in the $5^{\prime}$ splice site and in U6 snRNA has demonstrated a base-pair interaction between these RNAs at position +5 of the intron and further suggests that U6 snRNA can pair with additional nucleotides downstream of the +5 position (Kandels-Lewis and Seraphin 1993; Lesser and Guthrie 1993; Sun and Manley 1995).

The distal sequences in the $5^{\prime}$ splice site are therefore recognized at least twice during the splicing reaction, by the U1 and U6 snRNAs. The other nucleotides in the 5' splice site $(-1$ through +3$)$ may also be recognized a second time, probably in the binding of the U4/U5/U6 tri-snRNP. Formation of spliceosome-type complexes containing U2, U5, and U4/U6 snRNAs on short oligoribonucleotides comprising a $5^{\prime}$ splice site occurs when sequences at the $5^{\prime}$ terminus of U1 snRNA are blocked (Konforti et al. 1993; Konforti and Konarska 1994). In these experiments, nucleotides at positions -2 through +5 were critical for both efficient binding of the trisnRNP and for UV cross-linking of the U6 snRNA to the RNA oligo.

SR proteins, which contain extensive repeats of the dipeptide sequence serine-arginine, have been implicated in constitutive splicing and in the regulation of alternative splicing, such as the sex-determination pathway in Drosophila /Ge et al. 1991; Krainer et al. 1991; Fu et al. 1992; Zahler et al. 1992; Tian and Maniatis 1993; Lynch and Maniatis 1995). These proteins function during an early step in the commitment of a substrate to splicing (Fu 1993), in the formation of the E complex 
(Staknis and Reed 1994), and in bridging interactions between the $5^{\prime}$ splice site and the branch site (Wu and Maniatis 1993). Addition of SR proteins can switch the utilization of two competing 5' splice sites (Zahler et al. 1993; Zahler and Roth 1995), possibly by directly binding to 5' splice sites and recruiting U1 snRNP (Kohtz et al. 1994; Zuo and Manley 1994; Zahler and Roth 1995). SR proteins also participate in splicing by binding to exon splicing enhancers: sequences that stimulate adjacent splice site activity (Lavigueur et al. 1993; Sun et al. 1993; Tian and Maniatis 1993; Watakabe et al. 1993; Xu et al. 1993; Dirksen et al. 1994; Tanaka et al. 1994). SR proteins bound at these enhancers are postulated to promote U2 snRNP binding to the branch site, probably by recruiting and stabilizing the interaction of the branch site region with U2AF (Wang et al. 1995).

We demonstrated previously that SR proteins promote efficient splicing in extracts that have been depleted for U1 snRNP (Crispino et al. 1994). In a separate study, SR proteins were shown to reconstitute the splicing of premRNAs in extracts where the U1 snRNP was blocked by an antisense oligonucleotide (Tarn and Steitz 1994). SR proteins have also been demonstrated to promote the binding of U2 snRNP to the branch site of an RNA lacking a $5^{\prime}$ splice site (Tarn and Steitz 1995).

Here, we present evidence that splicing in U1 snRNPdepleted extracts supplemented with SR proteins has a rate-limiting step distinct from that in mock-depleted extracts. In Ul-independent reactions, a rate-limiting step is the base pairing of the pre-mRNA with U6 snRNA, probably during the binding of the U4/U5/U6 tri-snRNP. We also demonstrate that the entire $5^{\prime}$ splice site consensus sequence is recognized in the U1 snRNPdepleted reactions.

\section{Results \\ $S R$ proteins promote complex formation in \\ $U 1$ snRNP-depleted extracts in a \\ substrate-specific reaction}

The efficiency with which SR proteins complemented a U1 snRNP-depleted reaction was dependent on the particular pre-mRNA (Crispino et al. 1994). The $\beta$-globin pre-mRNA was processed in the U1 snRNP-depleted extract supplemented with SR proteins $(\Delta U 1+S R)$ to the same extent as in mock-depleted extracts, whereas an adenovirus pre-mRNA substrate, Ad1, was processed at $\sim 10 \%$ of the level observed in the mock-depleted extract. PIP85.A, a pre-mRNA with sequences based largely on Ad1, was not active for splicing in $\Delta \mathrm{U} 1+\mathrm{SR}$ reactions. To further analyze the nature of this substrate specificity, splicing reactions containing either the $\beta$-globin or PIP85.A pre-mRNAs were assayed for both splicing and spliceosome formation. As expected, the $\Delta \mathrm{U} 1+\mathrm{SR}$ reactions spliced $\beta$-globin to the same extent as the mock-depleted extract (Fig. 1A, cf. lanes 1 and 2 with 7 and 8), whereas the PIP85.A substrate was inactive (Fig. 1C, lanes 16-20).
Analysis of the reactions in Figure 1, A and C, by native gel electrophoresis (Konarska and Sharp 1986, 1987) revealed the predicted complexes in the mock-depleted extracts (Fig. 1, B and D, mock lanes). Complex A, composed of U2 snRNP stably associated with the branch site, and complex B, formed by the binding of the U4/ U5/U6 tri-snRNP to complex A, were rapidly generated. The slowest migrating complex, $\mathrm{C}$, which is produced by rearrangements within the spliceosome and the addition of other protein factors, was difficult to distinguish in these gels. Addition of SR proteins to mock-depleted extracts had little effect on complex formation or splicing (Fig. 1, A-D, mock + SR lanes).

U1 snRNP is required for the stable binding of U2 snRNP under normal conditions (Barabino et al. 1990). Consistent with this earlier finding, complex A formation was impaired on either pre-mRNA in the absence of U1 snRNP (Fig. 1, B and D, $\Delta$ U1 lanes). However, addition of high concentrations of SR proteins greatly stimulated the formation of complex A on the $\beta$-globin substrate in the U1 snRNP-depleted reactions (Fig. 1B, $\Delta \mathrm{U} 1+\mathrm{SR}$ lanes). The slower migrating $\mathrm{B}$ and $\mathrm{C}$ complexes were also present, consistent with the observed splicing activity in these reactions (Fig. 1A, $\Delta \mathrm{Ul}+\mathrm{SR}$ lanes). These complexes are less stable than those formed in mock-depleted extracts, as they were not detectable when reactions were electrophoresed at room temperature instead of at $4^{\circ} \mathrm{C}$ (data not shown). The addition of SR proteins slightly increased the amount of U2 snRNP bound to the PIP85.A substrate in reactions depleted of U1 snRNP (Fig. 1D, $\Delta U 1+$ SR lanes); this effect was minimal when compared with that observed with the $\beta$-globin substrate.

Thus, the difference between the splicing activities of the two substrates in the U1 snRNP-depleted/high SR conditions is reflected by the efficiency of formation of the U2 snRNP complex. SR proteins stimulated the transition from $\mathrm{H}$ complex to complex $\mathrm{A}$ in a substrate-specific manner. The identities of the complexes formed under the above conditions were confirmed by affinity selection experiments using biotinylated pre-mRNAs (data not shown). In addition, both pre-mRNAs failed to form detectable snRNP-bound complexes in the U2 snRNP-depleted extracts, consistent with the A complex being the earliest detectable species in this assay (data not shown).

\section{SC35 and SRp55 promote splicing in U1 snRNP-depleted extracts}

SR proteins derived from HeLa cells contain six major species: SRp75, SRp55, SRp40, ASF/SF2, SC35, and SRp20 (Zahler et al. 1992). To determine whether a single species can reconstitute activity, SC35 and SRp55, produced by baculovirus infection of insect cells, were assayed for their ability to restore splicing to U1 snRNPdepleted extracts (Fig. 2). The splicing of the $\beta$-globin pre-mRNA in mock-depleted and U1 snRNP-depleted 

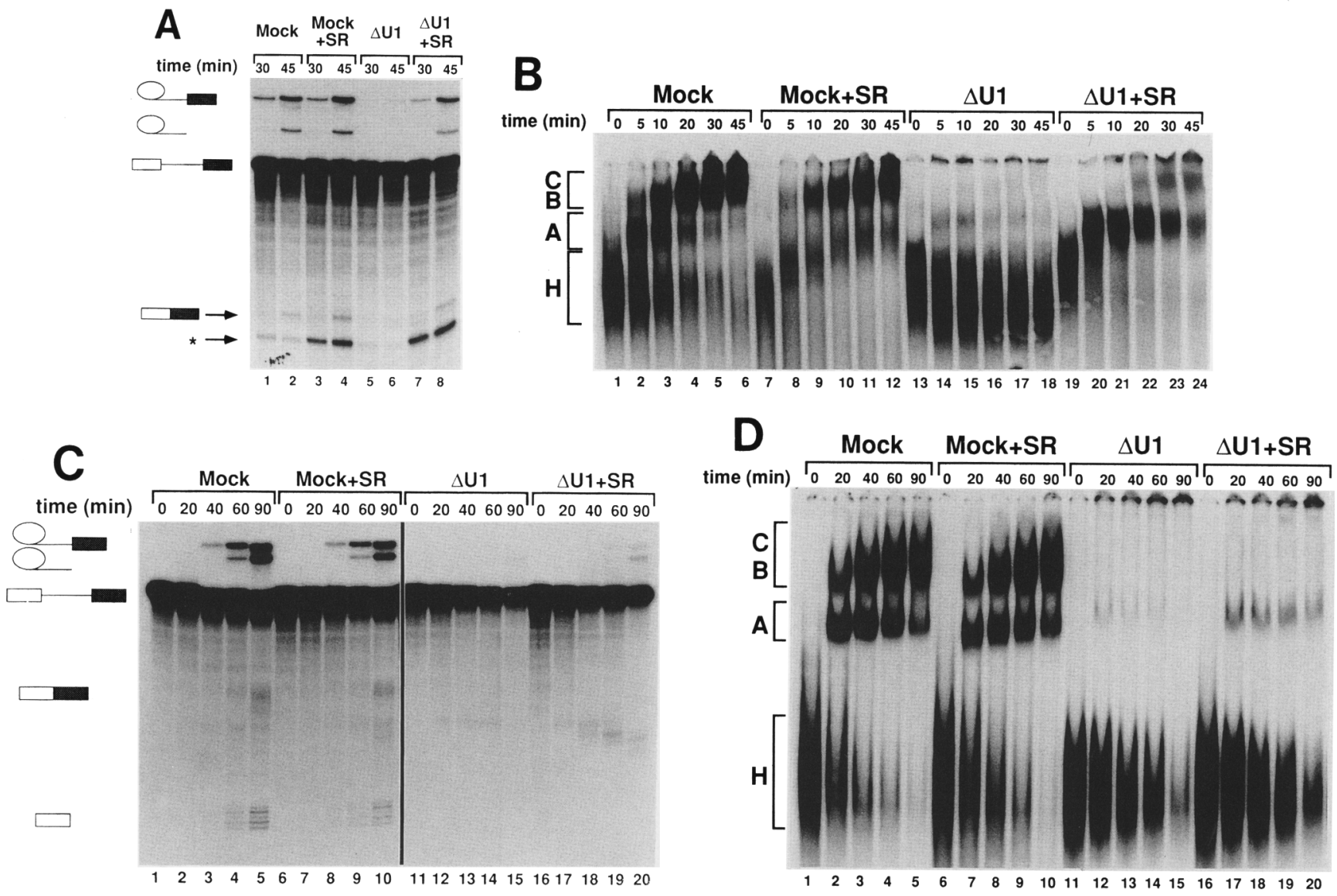

Figure 1. SR proteins promote complex A formation in reactions depleted of U1 snRNP. (A) Time course of splicing for $\beta$-globin pre-mRNA in mock and U1 snRNP-depleted extracts, supplemented with either $450 \mathrm{ng}$ of HeLa SR proteins or an equal volume of buffer. Splicing intermediates and products are identified by icons at left. The $5^{\prime}$ exon RNA is not shown, although it was detected in all active splicing lanes. The species marked with an asterisk $\left(^{*}\right)$ corresponds to a fragment protected by U2 snRNP from exonuclease digestion. $(B)$ Native gel analysis of the complexes formed on the $\beta$-globin pre-mRNA during the splicing reactions in $A$. The identities of the $\mathrm{H}, \mathrm{A}, \mathrm{B}$, and $\mathrm{C}$ complexes are indicated at left. $|C|$ Time course of splicing for PIP85.A pre-mRNA in mock and U1 snRNPdepleted extracts, supplemented with either $450 \mathrm{ng}$ of HeLa SR proteins or an equal volume of buffer. $(D)$ Native gel analysis of complexes formed on the PIP85.A substrate in the reactions of $C$.

extracts supplemented with different SR proteins was analyzed. Purified SC35 alone was sufficient to restore an equivalent level of splicing to the U1 snRNP-depleted extract as the HeLa SR proteins (Fig. 2, cf. lanes 4-6 with lane 3). Purified SRp55 also promoted splicing in the depleted extract, albeit with a reduced efficiency (lanes 7-9).

The activity of SC35 also parallels that of the complete SR preparation in its sequence specificity. Although SC35 was efficient in restoring splicing reactions with the $\beta$-globin RNA, it did not promote splicing of the PIP85.A substrate in U1 snRNP-depleted extracts and only weakly restored splicing to the Adl pre-mRNA (data not shown). SC35 also has the same activity in assays for complex formation as described for the SR proteins above (data not shown). To investigate the role of SR proteins in splicing in a more defined system, purified SC35 was used in all subsequent experiments.
The entire $5^{\prime}$ splice site is recognized in $U 1$ snRNP-depleted extracts supplemented with SC35

The sequences of the $5^{\prime}$ splice sites are conserved in mammalian introns with a consensus of AG/GUAAGU (the bar denotes the $5^{\prime}$ splice site). A subset of these nucleotides has been shown to be important in the binding of Ul and U6 snRNAs during the course of the splicing process (for review, see Madhani and Guthrie 1994). In reactions that lack U1 snRNP, one might predict that those nucleotides whose sole function is in the base pairing with U1 snRNA would be dispensable, whereas the bases that are involved in additional contacts would be required. To investigate the role of the 5 ' splice site during splicing in the absence of U1 snRNP, point mutations were introduced into the $\beta$-globin pre-mRNA at several positions. These mutants were then incubated in both mock and $\Delta \mathrm{U} 1+\mathrm{SC} 35$ (reconstituted) extracts. The 


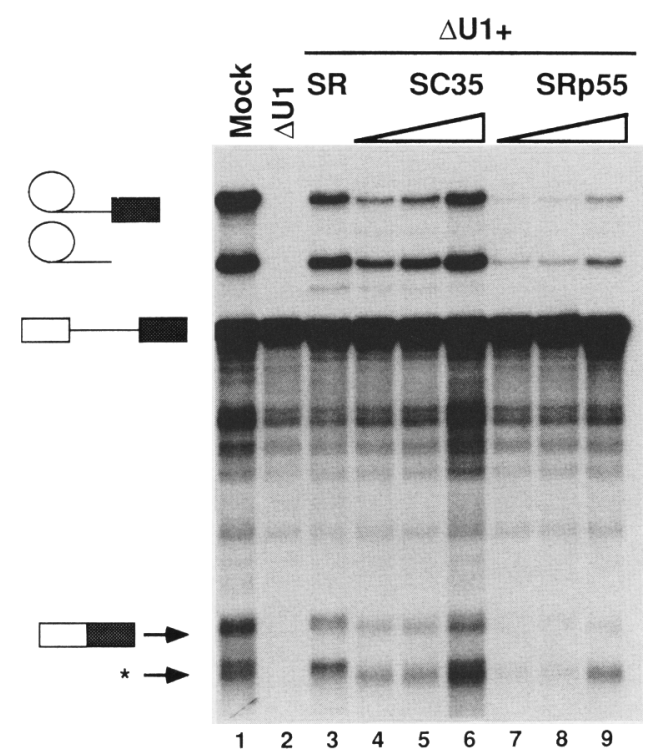

Figure 2. Purified SR proteins complement splicing extracts depleted for Ul snRNP. The $\beta$-globin pre-mRNA was incubated for $90 \mathrm{~min}$ in either mock or U1 snRNP-depleted nuclear extracts and supplemented with HeLa SR proteins ( $900 \mathrm{ng}$ ), purified SC35 $(100,300$, and $900 \mathrm{ng})$, or purified SRp55 $(100,300$, or $900 \mathrm{ng})$. The bands and icons are as described in Fig. 1.

rates of splicing in both reactions were determined and compared with the rates obtained with the wild-type premRNA (see Materials and methods).

For the wild-type substrate, the rates of appearance of splicing products in mock and reconstituted reactions were similar (Fig. 3A, cf. mock and $\Delta \mathrm{U} 1+$ SC35 splicing). Parallel splicing reactions with the $+1 \mathrm{G} \rightarrow \mathrm{A}$ mutant are shown. This mutation has been shown previously to delay 5 ' exon cleavage and to inhibit splicing by blocking the second step of the reaction (Lamond et al. 1987). In the mock-depleted extract, the RNA proceeded through the first step at $\sim 20 \%$ the rate of wild-type (Fig. 3A). Splicing of the equivalent step for this mutant in the reconstituted reactions was also reduced to $20 \%$ of wildtype. This indicates that the $G$ at the +1 position is critical for efficient splicing in both reaction types.

The rates of splicing for several other point mutations are summarized in Figure 3B. Positions $-1,+1,+2$, and +4 showed significantly reduced splicing rates in both mock and reconstituted reactions. Therefore, these nucleotides are important in both reaction pathways. In contrast, the +5 and +6 mutations had little effect on splicing in mock-depleted reactions but demonstrated a significant effect on splicing in the reconstituted reactions. Changes in the identity of the -2 position did not affect the rate of the first step in either pathway. This suggests that the nucleotides between the -1 and +2 and +4 through +6 positions are critical in a rate-limiting step of splicing in the U1 snRNP-depleted/SR supplemented reaction.

\section{Second-site mutations that increase potential pairing to U6 snRNA stimulate splicing only in the reconstituted reaction}

Several results suggest that the rate-limiting step for splicing in the reconstituted reactions is the binding of U6 snRNA to the distal sequences of the $5^{\prime}$ splice site. First, mutations in positions +5 and +6 , which are predicted to disrupt base pairing with U6 snRNA, have a stronger phenotype on splicing in reconstituted reac-
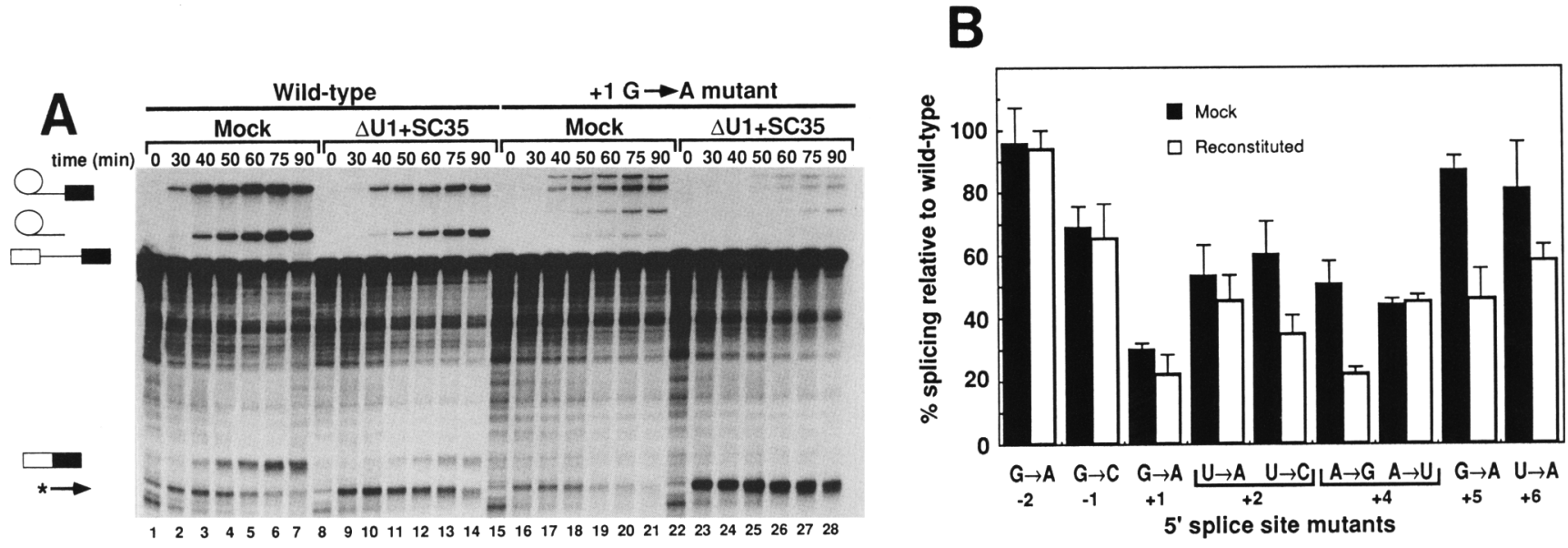

Figure 3. The $5^{\prime}$ splice site is recognized in the absence of Ul snRNP. (A) Time course of splicing of the wild-type and the $+1 \mathrm{G} \rightarrow \mathrm{A}$ point mutant in complete or reconstituted reactions. The complete reactions contained mock-depleted nuclear extract, whereas the reconstituted reactions were performed with U1 snRNP-depleted extract supplemented with 300 ng of SC35. The bands and icons are as described in Fig. 1. For the $+1 \mathrm{G} \rightarrow$ A point mutant, cryptic splice sites are utilized, leading to alternate forms of lariat intermediates and products. $(B)$ Graphical representation of the relative rates of the first step of splicing. Values are the average of three or more experiments with the error bars indicating 1 S.D. of the mean, except for the following mutants: $-2 \mathrm{G} \rightarrow \mathrm{A},+4 \mathrm{~A} \rightarrow \mathrm{U}$, and $+6 \mathrm{U} \rightarrow \mathrm{A}$ where the value represents the average of two experiments, with the error bars the range of the two determinations. 
tions than in normal reactions. Second, two different point mutations at the +4 position had different effects on the rate of splicing in the reconstituted reaction only. The $+4 \mathrm{~A} \rightarrow \mathrm{U}$ mutant spliced twofold more efficiently than the $+4 \mathrm{~A} \rightarrow \mathrm{G}$ mutant. According to the current model of U6 snRNA pairing to the 5' splice site (Fig. 4A), the adenosine at position 43 in U6 snRNA could basepair with the $+4 \mathrm{~A} \rightarrow \mathrm{U}$, increasing the stability of the helix and, hypothetically, the rate of splicing. This argument is qualified by the fact that the wild-type $\mathrm{A}$ at +4 would also not pair with position 43 of U6 snRNA but is more efficient than the $+4 \mathrm{U}$ mutation. This may suggest that this position is also recognized by another component of the spliceosome. Interestingly, the same allele specificity was observed in studies of the binding of the U4/U5/U6 tri-snRNP to a short oligoribonucleotide comprising the $5^{\prime}$ splice site (Konforti and Konarska 1994).

If the binding of U6 snRNA to the $5^{\prime}$ splice site is rate-limiting, then mutations that stabilize the base pairing of the 5' splice site with U6 snRNA might accelerate the rate of splicing in the reconstituted reactions. Previous studies have shown that positions $+7,+8$, and +9 of an intron can be recognized by base-pairing with U6 snRNA (Kandels-Lewis and Séraphin 1993; Lesser and Guthrie 1993). Positions +7 and +9 were changed to the complementary bases of the U6 snRNA nucleotides 38 and 40 in the context of the wild-type $\beta$-globin 5 ' splice

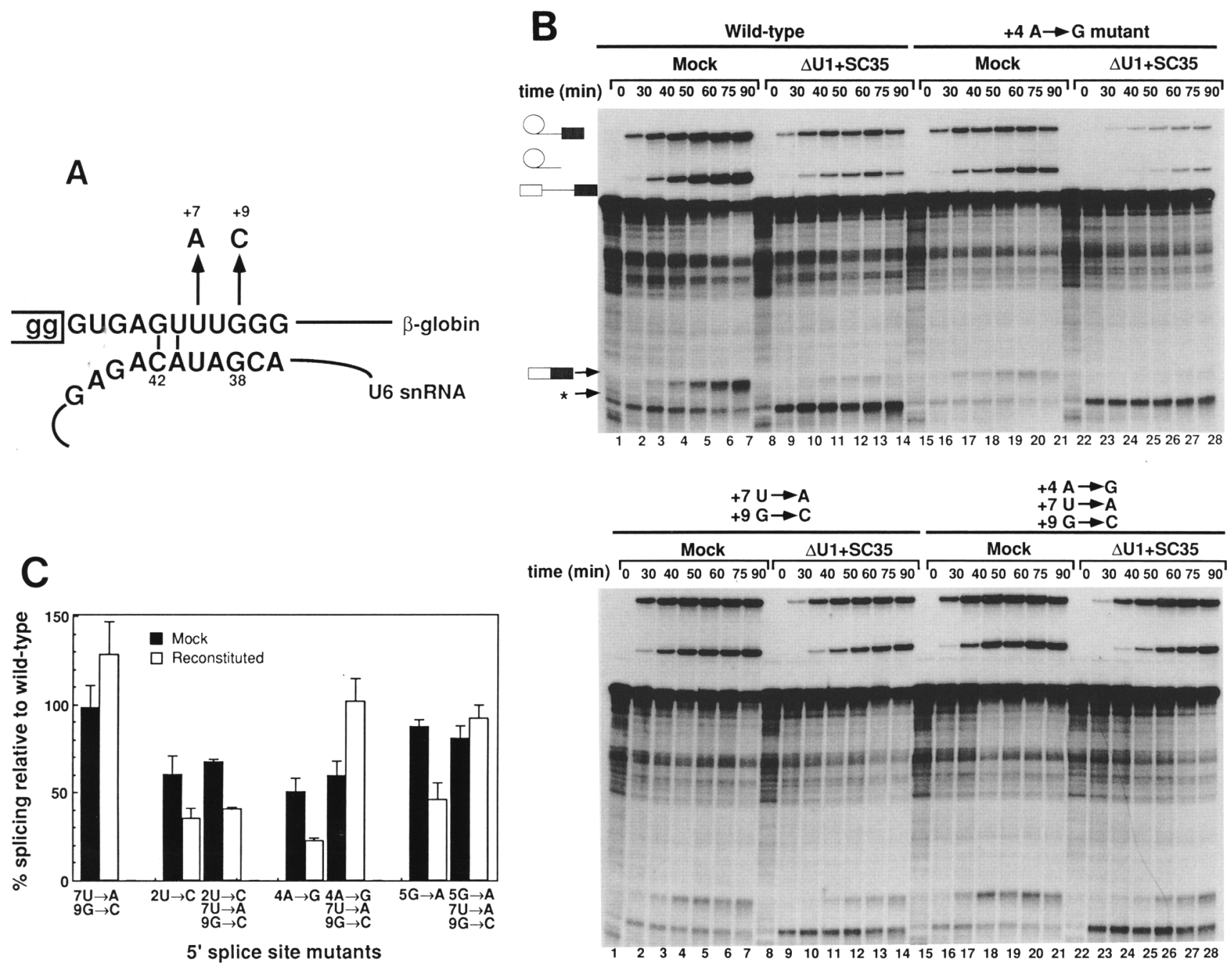

Figure 4. U6 snRNA binding is rate-limiting for splicing in the $\Delta U 1+S R$ reactions. $(A)$ Diagram of the $5^{\prime}$ splice site sequence of the $\beta$-globin pre-mRNA and the proposed pairing of the human U6 snRNA (for review, see Madhani and Guthrie 1994). The mutations introduced, which increase the complementarity between the $5^{\prime}$ splice site and the U6 snRNA, are shown. $(B)$ Splicing time courses of $\beta$-globin, the $+4 \mathrm{~A} \rightarrow \mathrm{G}$ mutant, the $+7 \mathrm{U} \rightarrow \mathrm{A} /+9 \mathrm{G} \rightarrow \mathrm{C}$ mutant, and the double mutant are shown. Purified SC35 was added to the Ul snRNP-depleted extract in the reconstituted reactions. The bands and icons are as described in Fig. 1 . (C) Graphical representation of the relative splicing rates for the $+2,+4$, and +5 mutants, along with the respective double mutants. Values are the average of three or more experiments, with the error bars indicating 1 S.D. of the mean, except for the following mutants: +2 $\mathrm{U} \rightarrow \mathrm{C} /+7 \mathrm{U} \rightarrow \mathrm{A} /+9 \mathrm{G} \rightarrow \mathrm{C}$ and $+5 \mathrm{G} \rightarrow \mathrm{A} /+7 \mathrm{U} \rightarrow \mathrm{A} /+9 \mathrm{G} \rightarrow \mathrm{C}$, where the values represent the average of two determinations, with the error bars the range of the two determinations. 
site and in each of three point mutants (Fig. 4A). The rates of splicing of these substrates were analyzed in mock reactions and in SR reconstituted reactions (Fig. 4C).

Splicing of the $+4 \mathrm{~A} \rightarrow \mathrm{G}$ and $+5 \mathrm{G} \rightarrow \mathrm{A}$ mutants was dramatically increased when the second-site mutations were introduced. An increase from $27 \%$ to $100 \%$ of wildtype splicing was observed in the reconstituted reactions for the $+4 \mathrm{~A} \rightarrow \mathrm{G}$ mutant, with little change in the splicing in mock extracts (Fig. 4B,C). Similarly, the splicing of the $+5 \mathrm{G} \rightarrow \mathrm{A}$ mutant increased from $37 \%$ of wild-type rate in the reconstituted reaction to $92 \%$ when the base pairing to U6 snRNA was increased. Again, the double mutants spliced to the same extent in the mock extracts.

In contrast, the $+2 \mathrm{U} \rightarrow \mathrm{C}$ mutant was not significantly rescued by the mutations that increased U6 pairing (Fig. 4C). Splicing was improved from $31 \%$ to $41 \%$, with no change in the splicing in mock extracts. Recognition of the $+2 \mathrm{U} \rightarrow \mathrm{C}$ mutation is apparently not limited by base pairing to U6 snRNA but by some other interaction.

U2-pre-mRNA complexes are formed in the reconstituted reactions prior to recognition of the 5' splice site

The above results suggest that association of the U4/U5/ U6 tri-snRNP can be rate-limiting in the reconstituted reaction. This binding depends on the presence of the U2 snRNP-pre-mRNA complex, which must have been available in nonlimiting amounts even in reactions with RNAs containing mutations in the $5^{\prime}$ splice site. In normal reactions, the association of the $5^{\prime}$ splice site with $\mathrm{U} 1$ snRNP is rate-limiting, and a delay in the rate of formation of the U2 snRNP complex can be detected for substrates with mutations in the $5^{\prime}$ splice site. These two results indicate that mutations in the $5^{\prime}$ splice site should affect formation of the U2 snRNP A complex differently under the two reaction conditions.
The rate of complex A formation with the $+1 \mathrm{G} \rightarrow \mathrm{A}$ mutation was compared with that of wild-type substrate, both in mock and $\Delta \mathrm{U} 1+\mathrm{SC} 35$ reactions (Fig. 5). As demonstrated previously for this mutant (Lamond et al. 1987), formation of the U2 snRNP complex A was greatly reduced compared with wild-type pre-mRNA in mock reactions. In contrast, for the same mutant, complex A formation in the reconstituted reactions was decreased only slightly from that for the wild-type substrates. The $+4 \mathrm{~A} \rightarrow \mathrm{G}$ mutant also showed reduced complex formation specifically in mock-depleted reactions (data not shown). These results indicate that the 5' splice site is recognized after U2 snRNP binding in U1 snRNP-depleted extracts supplemented with SR proteins and before $\mathrm{U} 2$ snRNP binding under complete reaction conditions.

\section{Discussion}

High concentrations of SR proteins circumvent the requirement for U1 snRNP in pre-mRNA splicing. This bypass reaction is substrate specific, probably reflecting the efficiency of binding of SR proteins. In this reaction, SR proteins first promote the rapid association of U2 snRNP with the branch site, in a manner independent of either U1 snRNP or the $5^{\prime}$ splice site (Fig. 6A). The entire $5^{\prime}$ splice site consensus sequence is required in a step after the formation of complex A (Fig. 6B). This recognition of the $5^{\prime}$ splice site occurs in a rate-limiting reaction with or after the binding of the U4/U5/U6 tri-snRNP. The distal nucleotides in the $5^{\prime}$ splice site are probably recognized by base pairing with U6 snRNA, whereas the proximal sequences interact with additional spliceosomal components.

\section{Base pairing with U6 snRNA is rate-limiting in U1 snRNP-depleted extracts}

The consensus sequence at the $5^{\prime}$ splice site was recognized in the U1 snRNP-depleted/SR reconstituted reac-

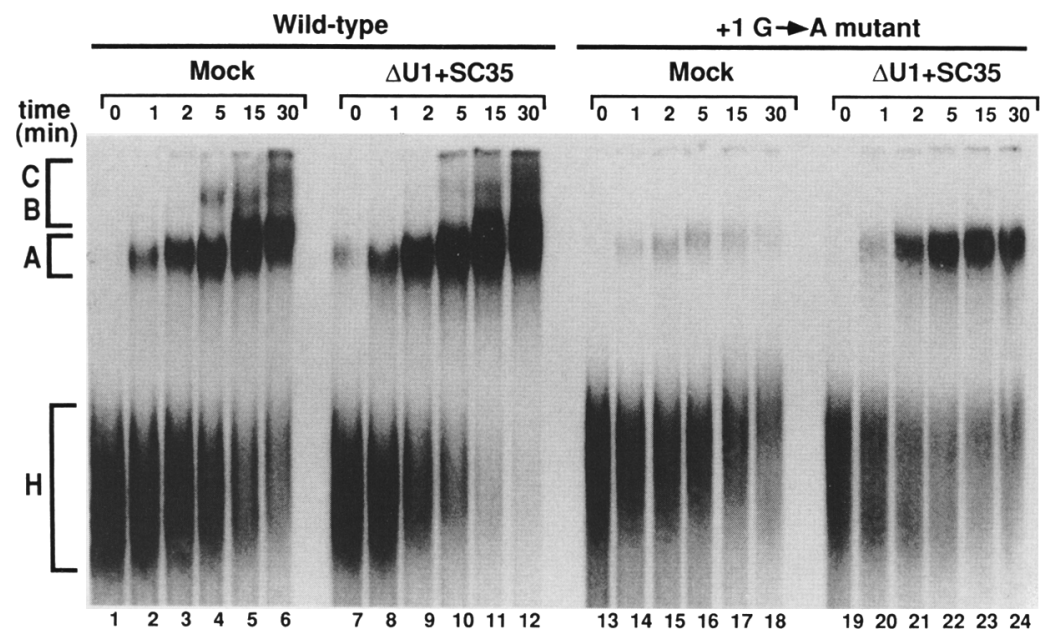

Figure 5. Native gel analysis of snRNP complexes formed on wild-type $\beta$-globin and the +1 $\mathrm{G} \rightarrow$ A mutant in the course of both complete and reconstituted splicing reactions. Reaction mixtures were treated with heparin prior to loading on the native polyacrylamide gel. 
Figure 6. (A) Proposed scheme for splicing in complete and SR reconstituted reactions. In complete reactions, U1 snRNP and SR proteins cooperatively interact to form E complex (Staknis and Reed 1994). U2 snRNP then binds to the branch site to produce complex A. In Ul-depleted extracts, excess SR proteins first form a complex with the pre-mRNA and then promote the association of U2 snRNP with the branch site. $(B)$ Interactions at the $5^{\prime}$ splice site during the course of splicing. In complete reactions, both $\mathrm{Ul}$ and U6 snRNAs base-pair to $5^{\prime}$ splice site sequences, and an additional factor, $X$, recognizes the proximal nucleotides in the $5^{\prime}$ splice site. In the SR reconstituted reactions, U6 snRNA and factor $\mathrm{X}$ bind to the 5' splice site in rate-limiting steps.
A

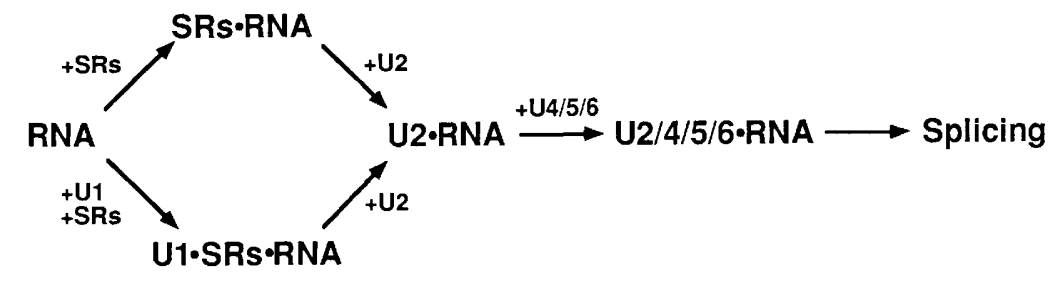

B

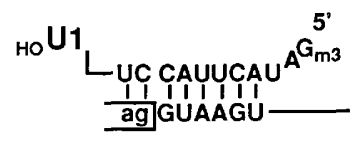

tions, because mutations in these sequences reduced the efficiency of splicing. This suggests that most of the positions between -1 and +6 of the intron are recognized by components other than U1 snRNP, probably U6 snRNA and other factors in the spliceosome (Fig. 6B). These results are consistent with previous experiments that characterized the binding of a spliceosometype complex lacking U1 snRNP to oligoribonucleotides containing the $5^{\prime}$ splice site sequence /Konforti and Konarska 1994). They are also consistent with the sequence specificity of trypanosome and nematode transsplicing, which does not apparently require an analog of U1 snRNP but does depend on the presence of the consensus sequence of the $5^{\prime}$ splice site (Nilsen 1993).

Previous evidence suggests that U6 snRNA binds to sequences $+4,+5$, and +6 of the $5^{\prime}$ splice site in the spliceosome (Kandels-Lewis and Séraphin 1993; Lesser and Guthrie 1993; Sun and Manley 1995). Disruption of these interactions could explain the phenotypes of $\mathrm{mu}$ tations in these positions in the absence of Ul snRNP. The proximal sequences, +1 and +2 of the $5^{\prime}$ splice site are also critical for efficient splicing in the absence of $U 1$ snRNA. These sequences must interact with other components of the spliceosome. One of these components is probably the p220 protein of the U4/U5/U6 particle, which cross-links to the base at the +2 position (J.L. Reyes and M.M. Konarska, pers. comm.).

Changes in the nucleotides at positions +4 and +5 reduced the efficiency of splicing in both the Ul snRNPdependent pathway and in the Ul snRNP-depleted/SR reconstituted pathway. The rates of splicing of these mutants were increased significantly in the reconstituted pathway, when additional changes at +7 and +9 that improved the base pairing of U6 snRNA to the substrate were introduced. However, the same changes did not increase the rate of splicing in the U1 snRNP-dependent pathway. This suggests that positions +4 and +5 were recognized by the U6 snRNA in a rate-limiting step of splicing in the former pathway but not in the latter. In the latter reaction, a step prior to the interaction with U6 snRNA is limited by mutations in the +4 and +5 positions, probably the U1 snRNP-dependent binding of $U 2$ snRNP.

It is interesting that mutations at the +2 position cannot be rescued by increasing sequence complementarity to U6 snRNA. This suggests that this position is recognized in a rate-limiting step other than the interaction with U6 snRNA, perhaps by a factor critical for the chemistry of the first step in splicing.

Although our data do not physically identify U6 snRNA binding as the rate-limiting event in U1 snRNPdepleted reactions supplemented with SR proteins, the combination of the mutant suppression results and the fact that efficient formation of complex A occurs under these conditions strongly implicates the interaction with U6 snRNA as rate-limiting for recognition of the $5^{\prime}$ splice site. The effects of the intron +7 and +9 mutational changes on splicing are most easily explained by the facilitation of the binding of U6 snRNA. Because these positions are not conserved among introns, it is unlikely that some uncharacterized factor binds specifically to these sequences and affects splicing in vitro. The only reasonable model is that U6 snRNA recognizes these positions in a potentially rate-limiting manner in the absence of Ul snRNP.

The data presented here give further strong evidence that the splicing reaction in the extracts depleted of $\mathrm{Ul}$ snRNP proceeds in a manner independent of this snRNP. The rate-limiting step in the depleted-reconstituted reaction was recognition of the $5^{\prime}$ splice site by U6 snRNA, whereas under normal conditions, the rate-limiting step is probably recognition of this sequence by Ul snRNP. Thus, recognition of the $5^{\prime}$ splice site in the Ul snRNPdepleted reactions is probably not mediated by contaminating levels of $\mathrm{U} 1 \mathrm{snRNP}$, as it is unlikely that reducing the level of U1 snRNP would shift the rate-limiting step to a subsequent step in the pathway. 
$S R$ proteins promote A complex formation in the $a b$ sence of U1 snRNP

The binding of U2 snRNP to pre-mRNAs usually occurs after the formation of the $\mathrm{E}$ complex, which contains Ul snRNP, U2AF, and SR proteins (Staknis and Reed 1994). However, in the absence of U1 snRNP, the addition of a threefold excess amount of SC35 is sufficient to form a stable U2 snRNP complex with the substrate. As would be anticipated from these observations, mutations in the $5^{\prime}$ splice site do not affect the rate of formation of the U2 snRNP complex in the presence of high amounts of SC35. This bypass reaction is sequence dependent, however, because it is not observed with the PIP85.A premRNA. This suggests that the SR proteins bypass a U1dependent step by promoting the binding of U2 snRNP to the branch site. SR proteins may also have a role in recruiting the U4/U5/U6 tri-snRNP to the U2 snRNPsubstrate complex as well (R.F. Roscigno and M.A. García-Blanco, pers. comm.; Tarn and Steitz 1995). The substrate specificity of this bypass reaction may reflect the potential differences in affinities of the SR proteins for the various substrates.

Preliminary studies with chimeric pre-mRNAs containing sequences from $\beta$-globin and PIP85.A have demonstrated that the $3^{\prime}$ portion of the $\beta$-globin RNA /comprising the branch site, polypyrimidine tract, and the $3^{\prime}$ exon), not the $5^{\prime}$ splice site region (upstream of the branch site), confers the ability to respond to the excess SR proteins (data not shown). Thus, SR proteins may act in U1 snRNP-depleted extracts by binding to a downstream sequence element and promoting the formation of A complex. The activity of SR proteins in the reconstituted reaction is reminiscent of that of exon splicing enhancer sequences. These sequence elements, which bind SR proteins, are thought to promote splicing of upstream introns by increasing the binding of U2AF to the branch site and thus assisting in U2 snRNP recruitment (Wang et al. 1995).

\section{The role of U1 snRNP in RNA splicing}

Ul snRNP was originally shown to be required for splicing by inhibiting its activity with either a specific antibody or cleavage with RNase $\mathrm{H}$ (for review, see Steitz et al. 1988). Several genetic studies subsequently demonstrated that the $5^{\prime}$ end of U1 snRNA associates with the 5 ' splice site by base pairing (Zhuang and Weiner 1986; Siliciano and Guthrie 1988). Some mutations of the $5^{\prime}$ splice site were suppressed by compensatory changes in U1 snRNA, but in many cases the compensatory changes did not restore splicing activity (Séraphin et al. 1988; Siliciano and Guthrie 1988; Séraphin and Rosbash 1990).

We now know that the same $5^{\prime}$ splice site sequences are recognized subsequent to U1 snRNA recognition, explaining this previously observed lack of complementation. These results are consistent with the hypothesis that U1 snRNP's primary role is to promote the assembly of U2 snRNP on the branch site, perhaps by facili- tating the binding of SR proteins to the substrate RNA The subsequent interaction of $S R$ proteins and the $70-\mathrm{kD}$ protein bound to the U1 snRNP with the SR factor U2AF could increase the rate of binding of U2 snRNP /Wu and Maniatis 1993; Kohtz et al. 1994). U1 snRNP is not, however, an important component of the spliceosome during execution of the chemistry of splicing.

In vivo, most pre-mRNAs are processed in the U1dependent manner, but there may exist alternative pathways for splicing. U1 snRNP could have a limited role in the splicing of some pre-mRNAs, specifically those that have a high affinity for SR proteins. In the nucleus, SR proteins are localized in speckles (Fu and Maniatis 1990; Blencowe et al. 1994). Splicing may occur within these speckles and on their periphery (Carter et al. 1993; Xing et al. 1993) where the concentration of SR proteins may be sufficiently high to promote splicing without U1 snRNP. This secondary splicing pathway would give the cell an additional way to regulate the splicing of different classes of pre-mRNAs.

\section{Materials and methods}

\section{Pre-mRNA substrates}

Point mutants $+1 \mathrm{G} \rightarrow \mathrm{A},+2 \mathrm{U} \rightarrow \mathrm{A}$, and $+2 \mathrm{U} \rightarrow \mathrm{C}$ are the previously characterized pBSAL 5 , pBSAL 6 , and pBSAL 7 plasmids, respectively (Lamond et al. 1987). The other mutant $\beta$-globin pre-mRNAs were constructed by PCR-based mutagenesis, and the final constructs were sequenced. Wild-type and mutant pre-mRNAs were transcribed using T3 RNA polymerase (Pharmacia).

\section{Preparation of snRNP-depleted nuclear extracts}

HeLa nuclear extracts were depleted using the antisense affinity selection method (Blencowe and Barabino 1995). The extracts were characterized extensively for activity and extent of depletion as described (Crispino et al. 1994).

\section{HeLa SR proteins}

SR proteins were prepared from HeLa cells and treated with micrococcal nuclease as described (Zahler at al. 1992; Crispino et al. 1994|. SC35 and SRp55 were overexpressed in Hi-5 cells and purified essentially as described (Fu 1993). These insect cell-derived proteins were treated with micrococcal nuclease in the same way as the HeLa cell preparation. The individual proteins generated single bands on both Coomassie-stained SDSpolyacrylamide gels and on Western blots probed with the $\mathrm{mAb}$ 104 monoclonal antibody (data not shown).

\section{Splicing reactions}

Splicing reactions were carried out under standard conditions using 20\% nuclear extract (Grabowski et al. 1984). SR proteins were added at levels indicated in the figure legends. For the native gel analysis in Figure 1, a fraction of the splicing reactions was loaded directly onto $4 \%$ polyacrylamide $(80: 1)$ gels that were electrophoresed in $1 \times$ TG $150 \mathrm{~mm}$ Tris-glycine at $\mathrm{pH}$ 8.8 . The remainder of the reactions were processed and analyzed on $6 \%$ polyacrylamide/ $8 \mathrm{M}$ urea $(19: 1)$ gels in $1 \times$ TBE 189 $\mathrm{mM}$ Tris-borate, $2 \mathrm{mM}$ EDTA/ to assay the extent of the splicing reactions. For the splicing complex analysis in Figure 5, the 
splicing reactions were treated with $0.5 \mathrm{mg} / \mathrm{ml}$ heparin for 5 min at $30^{\circ} \mathrm{C}$ prior to loading on the $4 \%$ polyacrylamide (80:1) gels. The native gels were electrophoresed either at $4^{\circ} \mathrm{C}$ (Fig. 1B) or at room temperature (Figs. ID and 5).

\section{Determination of relative splicing rates}

Polyacrylamide gels were quantitated using a Molecular Dynamics PhosphorImager and ImageQuant software version 3.22. The relative rates of the first step of splicing were determined essentially as described (Query et al. 1994). The relative amount of RNA in each band was quantitated and expressed as a percentage of the total obtained from summing the intermediates, products, and the precursor. For each band, an individual background value was determined from the region directly above that band. First step rates were determined from the linear portions of the curve and were calculated in such a way as to include all RNA species that had undergone the first step of splicing. The rates of splicing of the mutants were normalized, using parallel control reactions, to that of wild-type $\beta$-globin for each reaction type.

\section{Acknowledgments}

We thank X.-D. Fu for supplying the baculovirus clones that express individual SR proteins SC35 or SRp55. J. Kim was instrumental in purification of the individual SR proteins from Hi-5 cell lysates, and $\mathrm{R}$. Issner and $\mathrm{Y}$. Qiu are thanked for excellent technical assistance. B. Blencowe, A. MacMillan, J. Pomerantz, and C. Query kindly made helpful comments on the manuscript. Many thanks also to $M$. Siafaca for secretarial support. This work was supported by U.S. Public Health Service grants RO1-GM34277 and RO1-AI32486 from the National Institutes of Health and partially by a Cancer Center Support (core) grant, P30-CA14051, from the National Cancer Institute.

The publication costs of this article were defrayed in part by payment of page charges. This article must therefore be hereby marked "advertisement" in accordance with 18 USC section 1734 solely to indicate this fact.

\section{References}

Barabino, S.M.L., B.J. Blencowe, U. Ryder, B.S. Sproat, and A.I. Lamond. 1990. Targeted snRNP depletion reveals an additional role for mammalian U1 snRNP in spliceosome assembly. Cell 63: 293-302.

Blencowe, B.J. and S.M.L. Barabino. 1995. Antisense affinity depletion of RNP particles. In Methods in molecular biology, vol 37, In vitro transcription and translation protocols (ed. M.J. Tymms), pp. 67-76. Humana Press, Totowa, NJ.

Blencowe, B.J., J.A. Nickerson, R. Issner, S. Penman, and P.A. Sharp. 1994. Association of nuclear matrix antigens with exon-containing splicing complexes. J. Cell Biol. 127: 593607.

Carter, K.C., D. Bowman, W. Carrington, K. Fogarty, J.A. McNeil, F.S. Fay, and J.B. Lawrence. 1993. A three-dimensional view of precursor messenger RNA metabolism within the mammalian nucleus. Science 259: 1330-1335.

Crispino, J.D., B.J. Blencowe, and P.A. Sharp. 1994. Complementation by SR proteins of pre-mRNA splicing reactions depleted of U1 snRNP. Science 265: 1866-1869.

Dirksen, W.P., R.K. Hampson, Q. Sun, and F.M. Rottman. 1994. A purine-rich exon sequence enhances alternative splicing of bovine growth hormone pre-mRNA. I Biol. Chem. 269: 6431-6436.
Fu, X.-D. 1993. Specific commitment of different pre-mRNAs to splicing by single SR proteins. Nature 365: 82-85.

Fu, X.-D. and T. Maniatis. 1990. Factor required for mammalian spliceosome assembly is localized to discrete regions in the nucleus. Nature 343: 437-441.

Fu X.-D., A. Mayeda, T. Maniatis, and A.R. Krainer. 1992. General splicing factors SF2 and SC35 have equivalent activities in vitro, and both affect alternative $5^{\prime}$ and $3^{\prime}$ splice site selection. Proc. Natl. Acad. Sci. 89: 11224-11228.

Ge, H., P. Zuo, and J.L. Manley. 1991. Primary structure of the human splicing factor ASF reveals similarities with Drosophila regulators. Cell 66: $373-382$.

Grabowski, P.J., R.A. Padgett, and P.A. Sharp. 1984. Messenger RNA splicing in vitro: An excised intervening sequence and a potential intermediate. Cell 37: 415-427.

Kandels-Lewis, S. and B. Séraphin. 1993. Role of U6 snRNA in 5 ' splice site selection. Science 262: 2035-2039.

Kohtz, J.D., S.F. Jamison, C.L. Will, P. Zuo, R. Lührmann, M.A. García-Blanco, and J.L. Manley. 1994. Protein-protein interactions and $5^{\prime}$-splice-site recognition in mammalian mRNA precursors. Nature 368: 119-124.

Konarska, M.M. and P.A. Sharp. 1986. Electrophoretic separation of complexes involved in the splicing of precursors to mRNAs. Cell 46: 845-855.

- 1987. Interactions between small nuclear ribonucleoprotein particles in formation of spliceosomes. Cell 49: 763774.

Konforti, B.B. and M.M. Konarska. 1994. U4/U5/U6 snRNP recognizes the 5' splice site in the absence of U2 snRNP. Genes \& Dev. 8: 1962-1973.

Konforti, B.B., M.J. Koziolkiewicz, and M.M. Konarska. 1993. Disruption of base pairing between the $5^{\prime}$ splice site and the $5^{\prime}$ end of U1 snRNA is required for spliceosome assembly. Cell 75: 863-873.

Krainer, A.R., A. Mayeda, D. Kozak, and G. Binns. 1991. Functional expression of cloned human splicing factor SF2: Homology to RNA-binding proteins, $\mathrm{Ul} 70 \mathrm{~K}$, and Drosophila splicing regulators. Cell 66: 383-394.

Lamond, A.I., M.M. Konarska, and P.A. Sharp. 1987. A mutational analysis of spliceosome assembly: Evidence for splice site collaboration during spliceosome formation. Genes \& Dev. 1: 532-543.

Lavigueur, A., H. LaBranche, A.R. Kornblihtt, and B. Chabot. 1993. A splicing enhancer in the human fibronectin alternate ED1 exon interacts with SR proteins and stimulates U2 snRNP binding. Genes \& Dev. 7: 2405-2417.

Lesser, C.F. and C. Guthrie. 1993. Mutations in U6 snRNA that alter splice site specificity: Implications for the active site. Science 262: 1982-1988.

Lynch, K.W. and T. Maniatis. 1995. Synergistic interactions between two distinct elements of a regulated splicing enhancer. Genes \& Dev. 9: 284-293.

Madhani, H.D. and C. Guthrie. 1992. A novel base-pairing interaction between $\mathrm{U} 2$ and U6 snRNAs suggests a mechanism for catalytic activation of the spliceosome. Cell 71: 803-817.

- 1994. Dynamic RNA-RNA interactions in the spliceosome. Annu. Rev. Genet. 28: 1-26.

McPheeters, D.S. and J. Abelson. 1992. Mutational analysis of the yeast U2 snRNA suggests a structural similarity to the catalytic core of Group I introns. Cell 71: 819-831.

Michaud, S. and R. Reed. 1991. An ATP-independent complex commits pre-mRNA to the mammalian spliceosome assembly pathway. Genes \& Dev. 5: 2534-2546.

1993. A functional association between the $5^{\prime}$ and $3^{\prime}$ splice sites is established in the earliest prespliceosome 
complex (E) in mammals. Genes \& Dev. 7: 1008-1020.

Moore, M.J., C.C. Query, and P.A. Sharp. 1993. Splicing of precursors to messenger RNAs by the spliceosome. In The RNA world (ed. R.F. Gesterland and J.F. Atkins), pp. 303-357. Cold Spring Harbor Press, Cold Spring Harbor, NY.

Nilsen, T.W. 1993. Trans-splicing of nematode mRNA precursors. Annu. Rev. Microbiol. 47: 413-440.

Query, C.C., M.J. Moore, and P.A. Sharp. 1994. Branch nucleophile selection in pre-mRNA splicing: Evidence for the bulged duplex model. Genes \& Dev. 8: 587-597.

Sawa H. and J. Abelson. 1992. Evidence for a base-pairing interaction between U6 small nuclear RNA and 5' splice site during the splicing reaction in yeast. Proc. Natl. Acad. Sci. 89: 11269-11273.

Séraphin, B. and M. Rosbash. 1989. Identification of functional Ul snRNP-pre-mRNA complexes committed to spliceosome assembly and splicing. Cell 59: 349-358.

1990. Exon mutations uncouple 5 ' splice site selection from Ul snRNA pairing. Cell 63: 619-629.

Séraphin, B., L. Kretzner, and M. Rosbash. 1988. A U1 snRNP: pre-mRNA base pairing interaction is required early in yeast spliceosome assembly, but does not uniquely define the $5^{\prime}$ cleavage site. EMBO J. 7: 2533-2538.

Siliciano, P.G. and C. Guthrie. 1988. 5' splice site selection in yeast: Genetic alterations in base-pairing with Ul reveal additional requirements. Genes \& Dev. 2: 1258-1267.

Sontheimer, E. and J.A. Steitz. 1993. The U5 and U6 small nuclear RNAs as active site components of the spliceosome. Science 262: 1989-1996.

Staknis, D. and R. Reed. 1994. SR proteins promote the first specific recognition of pre-mRNA and are present together with U1 small ribonucleoprotein particle in a general splicing enhancer complex. Mol. Cell. Biol. 14: 7670-7682.

Steitz, J.A., D.L. Black, V. Gerke, K.A. Parker, A. Krämer, D. Frendewey, and W. Keller. 1988. Functions of the abundant U-snRNPs. In Structure and function of major and minor small ribonucleoprotein particles (ed. M.L. Birnsteil), pp. 115-154. Springer-Verlag, New York.

Sun, Q. and J. Manley. 1995. A novel U2-U6 snRNA structure is necessary for mammalian mRNA splicing. Genes \& Dev. 9: 843-854.

Sun, Q., A. Mayeda, R.K. Hampson, A.R. Krainer, and F.R. Rottman. 1993. General splicing factor SF2/ASF promotes alternative splicing by binding to an exonic splicing enhancer. Genes \& Dev. 7: 2598-2608.

Tanaka, K., A. Watakabe, and Y. Shimura. 1994. Polypurine sequences within a downstream exon function as a splicing enhancer. Mol. Cell Biol. 14: 1347-1354.

Tarn, W.-Y. and J.A. Steitz. 1994. SR proteins can compensate for the loss of U1 snRNP functions in vitro. Genes \& Dev. 8: 2704-2717.

1995. Modulation of 5' splice site choice in pre-messenger RNA by two distinct steps. Proc. Natl. Acad. Sci. 92: 2504-2508.

Tian, M. and T. Maniatis. 1993. A splicing enhancer complex controls alternative splicing of doublesex pre-mRNA. Cell 74: 105-114.

Wang, Z., H.M. Hoffmann, and P.J. Grabowski. 1995. Intrinsic U2AF binding is modulated by exon enhancer signals in parallel with changes in splicing activity. RNA 1: 21-35.

Watakabe, A., K. Tanaka, and Y. Shimura. 1993. The role of exon sequences in splice site selection. Genes \& Dev. 7: 407-418.

Wu, J.Y. and T. Maniatis. 1993. Specific interactions between proteins implicated in splice site selection and regulated alternative splicing. Cell 75: 1061-1070.
Xing, Y., C.V. Johnson, P.R. Dobner, and J.B. Lawrence. 1993. Higher level organization of individual gene transcription and RNA splicing. Science 259: 1326-1330.

$\mathrm{Xu}, \mathrm{R} ., \mathrm{J}$. Teng, and T.A. Cooper. 1993. The cardiac troponin T alternative exon contains a novel purine-rich positive splicing element. Mol. Cell. Biol. 13: 3660-3674.

Zahler, A.M. and M.B. Roth. 1995. Distinct functions of SR proteins in recruitment of U1 small ribonucleoprotein to alternative 5' splice sites. Proc. Natl. Acad. Sci. 92: 26422646.

Zahler, A.M., W.S. Lane, J.A. Stolk, and M.B. Roth. 1992. SR proteins: A conserved family of pre-mRNA splicing factors. Genes \& Dev. 6: 837-847.

Zahler, A.M., K.M. Neugebauer, W.S. Lane, and M.B. Roth. 1993. Distinct functions of SR proteins in alternative premRNA splicing. Science 260: 219-222.

Zhuang Y. and A.W. Weiner. 1986. A compensatory base change in U1 snRNA suppresses a $5^{\prime}$ splice site mutation. Cell 46: $827-835$.

Zuo, P. and J.L. Manley. 1994. The human splicing factor ASF/ SF2 can specifically recognize pre-mRNA 5 ' splice sites. Proc. Nat1. Acad. Sci. 91: 3363-3367. 


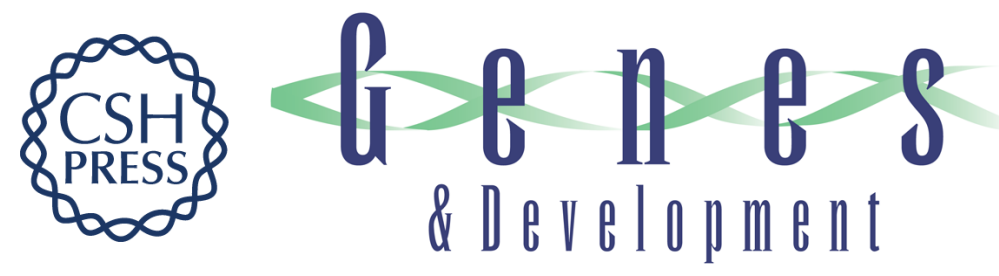

\section{A U6 snRNA:pre-mRNA interaction can be rate-limiting for U1-independent splicing.}

J D Crispino and P A Sharp

Genes Dev. 1995, 9:

Access the most recent version at doi:10.1101/gad.9.18.2314

References This article cites 51 articles, 31 of which can be accessed free at:

http://genesdev.cshlp.org/content/9/18/2314.full.html\#ref-list-1

License

Email Alerting

Service

Receive free email alerts when new articles cite this article - sign up in the box at the top right corner of the article or click here.

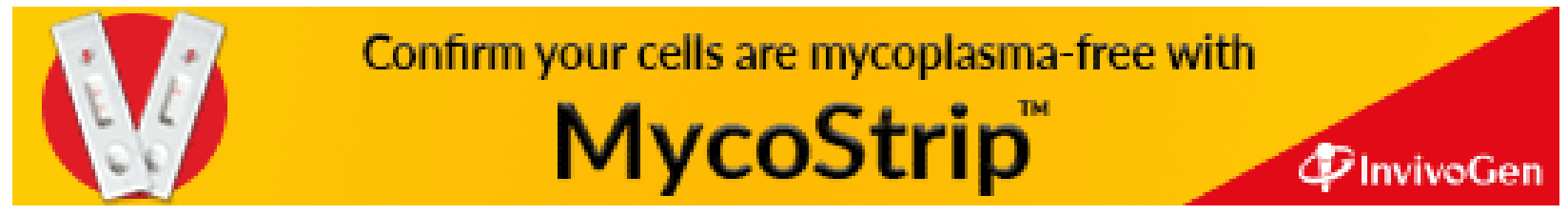

